Bull, Korean Math. Soc. 45 (2008), No. 3, pp. 457-466

\title{
A KUROSH-AMITSUR LEFT JACOBSON RADICAL FOR RIGHT NEAR-RINGS
}

\author{
Ravi Srinivasa Rao and K. Siva Prasad
}

\begin{abstract}
Let $R$ be a right near-ring. An $R$-group of type-5/2 which is a natural generalization of an irreducible (ring) module is introduced in near-rings. An $R$-group of type-5/2 is an $R$-group of type- 2 and an $R$-group of type-3 is an $R$-group of type-5/2. Using it $J_{5 / 2}$, the Jacobson radical of type-5/2, is introduced in near-rings and it is observed that $\mathrm{J}_{2}(R) \subseteq \mathrm{J}_{5 / 2}(R) \subseteq \mathrm{J}_{3}(R)$. It is shown that $\mathrm{J}_{5 / 2}$ is an ideal-hereditary Kurosh-Amitsur radical (KA-radical) in the class of all zero-symmetric near-rings. But $\mathrm{J}_{5 / 2}$ is not a KA-radical in the class of all near-rings. By introducing an $R$-group of type- $(5 / 2)(0)$ it is shown that $J_{(5 / 2)(0)}$, the corresponding Jacobson radical of type- $(5 / 2)(0)$, is a KA-radical in the class of all near-rings which extends the radical $\mathrm{J}_{5 / 2}$ of zero-symmetric near-rings to the class of all near-rings.
\end{abstract}

\section{Introduction}

Near-rings considered are right near-rings and $R$ stands for a right nearring. Many generalizations of the Jacobson radical of rings to near-rings were introduced and studied. Let $\nu \in\{0,1,2\}$. $\mathrm{J}_{\nu}$, the Jacobson radical of type$\nu$, was introduced and studied by Betsch [1] and $\mathbf{J}_{3}$, the Jacobson radical of type-3, was introduced and studied by Holcombe [2]. In this paper an $R$-group of type-5/2 is introduced as a natural generalization of an irreducible (ring) module. The corresponding Jacobson radical $J_{5 / 2}$ is also introduced in nearrings. Moreover, $\mathrm{J}_{2}(R) \subseteq \mathrm{J}_{5 / 2}(R) \subseteq \mathrm{J}_{3}(R)$. $\mathrm{J}_{5 / 2}$ is an ideal-hereditary KuroshAmitsur radical (KA-radical) in the class of all zero-symmetric near-rings. But $\mathrm{J}_{5 / 2}$ is not a KA-radical in the class of all near-rings. By introducing an $R$-group of type- $(5 / 2)(0)$ it is proved that $\mathrm{J}_{(5 / 2)(0)}$, the corresponding Jacobson radical of type-(5/2) $(0)$, is a KA-radical in the class of all near-rings which extends the radical $J_{5 / 2}$ of zero-symmetric near-rings to the class of all near-rings.

We recall some of the definitions related to $R$-groups and Jacobson radicals of near-rings.

Let $G$ be an $R$-group and $R_{0}$ be the zero-symmetric part of $R$. Then $G$ is

Received April 27, 2007.

2000 Mathematics Subject Classification. $16 \mathrm{Y} 30$.

Key words and phrases. near-ring, $R$-groups of type-5/2 and $(5 / 2)(0)$, Jacobson radicals of type-5/2 and $(5 / 2)(0)$. 
(i) monogenic if there is a $g \in G$ such that $R g=G$.

(ii) strongly monogenic if $G$ is monogenic and for each $g \in G$ either $R g=0$ or $G$.

(iii) an $R$-group of type- 0 if $G \neq 0$ and is a monogenic simple $R$-group.

(iv) an $R$-group of type- 1 if $G$ is of type- 0 and strongly monogenic.

(v) an $R$-group of type- 2 if $G \neq 0$, monogenic and $R_{0}$-simple.

(vi) an $R$-group of type-3 if $G$ is an $R$-group of type-2 and $x, y \in G$ and $r x=r y$ for all $r \in R$ implies $x=y$.

If $I$ is an ideal of $R$, then it is denoted by $I \triangleleft R$.

Let $Q$ be a mapping which assigns to each near-ring $R$ an ideal $Q(R)$ of $R$. Such mappings are called ideal-mappings. We consider the following properties which $Q$ may satisfy:

(H1) $h(Q(R)) \subseteq Q(h(R))$ for all homomorphisms $h$ of $R$;

(H2) $Q(R / Q(R))=\{0\}$ for all $R$;

$Q$ is $r$-hereditary if $I \cap Q(R) \subseteq Q(I)$ for all ideal $I$ of $R$;

$Q$ is $s$-hereditary if $Q(I) \subseteq I \cap Q(R)$ for all ideals $I$ of $R$;

$Q$ is ideal-hereditary if it is both $r$-hereditary and $s$-hereditary, that is, if $Q(I)=I \cap Q(R)$ for all ideals $I$ of $R$;

$Q$ is idempotent if $Q(Q(R))=Q(R)$ for all $R$;

$Q$ is complete if $Q(I)=I$ and $I$ is an ideal of $R$ implies $I \subseteq \mathrm{Q}(R)$.

With $Q$ we associate two classes of near-rings $\mathbb{R}_{Q}$ and $\mathbb{S}_{Q}$ defined by $\mathbb{R}_{Q}:=$ $\{R \mid Q(R)=R\}, \mathbb{S}_{Q}:=\{R \mid Q(R)=0\}$ and are called $Q$-radical class and - $Q$-semisimple class respectively.

- An ideal-mapping $Q$ is a Hoehnke radical (H-radical) if it satisfies conditions (H1) and (H2).

- An ideal-mapping $Q$ is a Kurosh-Amitsur radical (KA-radical) if it is a complete idempotent H-radical.

Let $\mathbb{M}$ be a class of near-ring. Classes of near-rings always assumed to be abstract, that is, they contains the one element near-ring and are closed under isomorphic copies. With every near-ring $R$, we associate two ideals of $R$, depending on $\mathbb{M}$. These ideals are defined by:

$\mathbb{M}(R):=\Sigma\{I \mid I$ is an ideal of $R$ and $I \in \mathbb{M}\}$ and

$(R) \mathbb{M}:=\cap\{I \mid I$ is an ideal of $R$ and $R / I \in \mathbb{M}\}$.

$\mathbb{M}$ is called regular if $0 \neq I \triangleleft R \in \mathbb{M}$ implies that $0 \neq I / K \in \mathbb{M}$ for some $K$ $\checkmark I$; hereditary if $I \triangleleft R \in \mathbb{M}$ implies $I \in \mathbb{M}$ and; c-hereditary if $I$ is a left invariant ideal of $R \in \mathbb{M}$, then $I \in \mathbb{M}$. (An ideal $I$ of $R$ is left invariant if $R I$ $\subseteq I$.)

A class of near-rings $M$ is a Kurosh-Amitsur radical class (KA-radical class) if it satisfies the following:

(R1) $M$ is closed under homomorphic images;

(R2) $\mathbb{M}(R) \in \mathbb{M}$ for all near-rings $R$;

(R3) $\mathbb{M}(R / \mathbb{M}(R))=\{0\}$ for all near-rings $R$. 
With a KA-radical class $\mathbb{R}$ we associate its semisimple class $\mathcal{S} \mathbb{R}:=\{R \mid \mathbb{R}(R)=$ $\{0\}\}$.

The following properties for a KA-radical class $\mathbb{R}$ are well known.

(i) $\mathbb{R}$ is hereditary if and only if $\mathbb{R}(R) \cap I \subseteq \mathbb{R}(\mathrm{I})$ for all $I \triangleleft R$.

(ii) $\mathcal{S} \mathbb{R}$ is hereditary if and only if $\mathbb{R}(\mathrm{I}) \subseteq \mathbb{R}(R) \cap I$ for all $I \triangleleft R$.

(iii) $\mathbb{R}$ is c-hereditary if and only if $\mathbb{R}(R) \cap I \subseteq \mathbb{R}(\mathrm{I})$ for all left invariant ideals $I$ of $R$.

We say that a class $M$ of near-rings satisfy condition $\left(\mathrm{F}_{l}\right)$ if $K \triangleleft I$ and $I$ is a left invariant ideal of $R$ with $I / K \in \mathbb{M}$, then $K \triangleleft R$.

Theorem 1.1 (Corollary 2.3 of [5]). Let $\mathbb{M}$ be a class of zero-symmetric nearrings and $\mathcal{L}$ be defined by $\mathcal{L}(R):=(R) \mathrm{M}$ and $\mathcal{L}_{0}$ be the restriction of $\mathcal{L}$ to the class of all zero-symmetric near-rings. Then the following are equivalent.

(1) $\mathcal{L}$ is a KA-radical in the class of all near-rings with $\mathcal{L}(I) \subseteq \mathcal{L} \cap I$ for all $I \triangleleft R$ and equality holds if $I$ is left invariant.

(2) $\mathcal{L}_{\circ}$ is an ideal-hereditary KA-radical in the class of all zero-symmetric near-rings and $\mathbb{M}$ satisfies condition ( $*$ ):

(*) If $K \triangleleft I \triangleleft R$ with $I$ a left invariant ideal of $R$ and $I / K \in \mathbb{M}$, then $\overline{R_{c}} \subseteq K$, where $\overline{R_{c}}$ is the ideal of $R$ generated by the subnear-ring $R_{c}$.

Theorem 1.2 (Theorem 4.2 .3 of [5]). The class of all zero-symmetric 2primitive near-rings satisfy condition $\left(F_{l}\right)$.

\section{2. $R$-groups of type-5/2}

Throughout this section $R$ stands for a right near-ring.

Definition 2.1. Let $G$ be an $R$-group. Then $G$ is called an $R$-group of type-5/2 if $G$ is an $R$-group of type- 2 and $R g=G$ for all $0 \neq g \in \mathrm{G}$.

Remark 2.2. From the definition we have that an $R$-group of type-5/2 is an $R$-group of type- 2 .

Proposition 2.3. An R-group of type-3 is an R-group of type-5/2.

Proof. Let $G$ be an $R$-group of type-3. So, $G$ is an $R$-group of type-2. Let 0 $\neq g \in G$. Since $G$ is an $R$-group of type-2, it is an $R$-group of type-1. So, either $R g=G$ or $R g=\{0\}$. Suppose that $R g=0$. Now $R 0=R_{c} 0=R_{c} g \subseteq$ $R g=0$ and hence $R 0=\{0\}$. So, $r g=r 0$ for all $r \in R$. Since $G$ is an $R$-group of type-3, $g=0$. This is a contradiction to the fact that $g \neq 0$. Therefore, $R g=G$.

Proposition 2.4. Let $R$ be a zero-symmetric near-ring and $\{0\} \neq G$ be an $R$-group. Then $G$ is an $R$-group of type-5/2 if and only if $R g=G$ for all $0 \neq$ $g \in G$. 
Proof. If $G$ is an $R$-group of type-5/2, then obviously $R g=G$ for all $0 \neq g \in$ $G$. Suppose that $R g=G$ for all $0 \neq g \in G$. Let $\{0\} \neq H$ be an $R$-subgroup of $G$. Let $0 \neq h \in H$. Now $G=R h \subseteq H$ and hence $H=G$. Therefore, $G$ is an $R$-group of type-2 and hence it is an $R$-group of type- $5 / 2$.

We present an example of an $R$-group of type- $5 / 2$ which is not an $R$-group of type-3.

Example 2.5. Let $(R,+)$ be a group of order $\geq 3$. Let $a, b \in R$. Define $a b=a$ if $b \neq 0$ and $a b=0$ if $b=0$. Now $R$ is a zero-symmetric near-ring. Moreover, $R a=R$ for all $0 \neq a \in R$. Therefore, by Proposition 2.4, $R$ is an $R$-group of type-5/2. Let $0 \neq b, 0 \neq c \in R$ and $b \neq c$. Now $a b=a=a c$ for all $a \in R$. So, $R$ is not an $R$-group of type- 3 .

Now we give an example of an $R$-group of type- 2 which is not an $R$-group of type-5/2.

Example 2.6. Let $(R,+)$ be a group of order $\geq 3$. Let $S$ be a non-empty subset of $R \backslash\{0\}$ such that $R \backslash \mathrm{S}$ contains no non-zero subgroup of $(R,+)$. Let $a, b \in R$. Define $a b=a$ if $b \in S$ and $a b=0$ if $b \notin S$. Now $R$ is a zero-symmetric near-ring. We have that $R b=\{0\}$ if $b \notin S$ and $R b=R$ if $b \in S$. Now it is clear that $R$ is an $R$-group of type- 2 . But, by Proposition $2.4, R$ is not an $R$-group of type- $5 / 2$.

Definition 2.7. A modular left ideal $L$ of $R$ is said to be a 5/2-modular left ideal of $R$ if $R / L$ is an $R$-group of type- $5 / 2$.

Proposition 2.8. Let $G$ be an $R$-group of type-5/2 and $0 \neq g \in G$. Then $(0: g)$ is a 5/2-modular left ideal of $R$ and $R /(0: g)$ and $G$ are isomorphic $R$-groups.

Proof. The mapping $h: R \rightarrow G$ defined by $h(r)=r g$ is an $R$-homomorphism of $R$ onto $G$ with Ker $h=(0: g)$ which is a modular left ideal of $R$. Now $R /(0: g)$ is isomorphic to $G$ as $R$-groups. So, $(0: g)$ is a $5 / 2$-modular left ideal of $R$.

Definition 2.9. $R$ is called a 5/2-primitive near-ring if $R$ has a faithful $R$ group of type-5/2.

Definition 2.10. An ideal $I$ of $R$ is called a 5/2-primitive ideal of $R$ if $R / I$ is a $5 / 2$-primitive near-ring.

One can easily verify the following.

Proposition 2.11. Let $I$ be an ideal of $R$. Then 
(1) If $G$ is an $R$-group of type-5/2 and $I \subseteq(0: G)$, then $G$ is also an $R / I$-group of type-5/2, where $(r+I) g:=r g, r+I \in R / I$ and $g \in G$. If in addition $I=(0: G)$, then $G$ is a faithful $R / I$-group.

(2) If $G$ is an $R / I$ group of type-5/2, then $G$ is also an $R$-group of type$5 / 2$, where $r g:=(r+I) g, r \in R$ and $g \in G$. If in addition $G$ is a faithful $R / I$-group, then $I=(0: G)_{R}$.

An immediate consequence of Propositions 2.8 and 2.11 is the following.

Proposition 2.12. Let $I$ be an ideal of $R$. Then the following are equivalent.

(i) $I$ is a 5/2-primitive ideal of $R$.

(ii) $I=(0: G)$ for some $R$-group $G$ of type-5/2.

(iii) $I=(L: R)$ for some $5 / 2$-modular left ideal $L$ of $R$.

Corollary 2.13. The following are equivalent

(i) $\{0\}$ is a 5/2-primitive ideal of $R$.

(ii) $R$ is 5/2-primitive.

(iii) $R$ has a 5/2-modular left ideal $L$ such that $(L: R)=\{0\}$.

We know that an ideal $P$ of $R$ is a 3-prime ideal of $R$ if $a, b \in R$ and $a R b \subseteq$ $P$ implies $a \in P$ or $b \in P$.

Proposition 2.14. Let $P$ be a 5/2-primitive ideal of $R$. Then $P$ is a 3-prime ideal of $R$.

Proof. Let $P$ be a 5/2-primitive ideal of $R$. We get an $R$-group $G$ such that $P=(0: G)$. Let $a, b \in R$ and $a R b \subseteq P=(0: G)$. Suppose that $b \notin P$. Now $b g \neq 0$ for some $g \in G$. So $R(b g)=G$ as $G$ is an $R$-group of type-5/2. Therefore, $a G=a R(b g)=(a R b) g=\{0\}$. So $a \in(0: G)=P$. Hence $P$ is 3-prime.

We know that a 3-primitive ideal of a zero-symmetric near-ring is equiprime and 3 -prime. So with the introduction of $5 / 2$-primitive ideals, we have primitive ideals which are 3 -prime but not equiprime.

\section{The Jacobson radical of type-5/2}

Definition 3.1. The Jacobson radical of $R$ of type-5/2, denoted by $\mathrm{J}_{5 / 2}(R)$, is defined as the intersection of all 5/2-primitive ideals of $R$ and if $R$ has no such ideals, then $\mathrm{J}_{5 / 2}(R)$ is defined as $R$.

Remark 3.2. By Proposition 2.12, $\mathrm{J}_{5 / 2}(R)=\cap\{(0: G) \mid G$ is an $R$-group of type- $5 / 2\}=\cap\{(L: R) \mid L$ is a $5 / 2$-modular left ideal of $R\}$.

The following proposition is immediate.

Proposition 3.3. $J_{5 / 2}(R)=\cap\{P \mid R / P$ is a 5/2-primitive near-ring $\}$.

Proposition 3.4. $J_{5 / 2}(R)=\cap\{L \mid L$ is a 5/2-modular left ideal of $R\}$. 
Proof. If $R$ has no 5/2-primitive ideals, then by Proposition 2.12, $R$ has no $5 / 2$-modular left ideals. So, if $\mathrm{J}_{5 / 2}(R)=R$, then the result follows. Now suppose that $R$ has a $5 / 2$-primitive ideal. So there is an $R$-group of type-5/2. We have $\mathrm{J}_{5 / 2}(R)=\cap\{(0: G) \mid G$ is an $R$-group of type-5/2 $\}$. Let $G$ be an $R$-group of type-5/2. Let $0 \neq g \in G$. Since $R g=G$, we get that $r \rightarrow \mathrm{rg}$ is an $R$-homomorphism of $R$ onto $G$ with Kernel $(0: g)$. So $R /(0: g)$ and $G$ are isomorphic $R$-groups and hence $(0: g)$ is a $5 / 2$-modular left ideal of $R$. Therefore $(0: G)$ is an intersection of $5 / 2$-modular left ideals of $R$. This shows that $\mathrm{J}_{5 / 2}(R)$ is an intersection of $5 / 2$-modular left ideals of $R$. Let $T$ be a $5 / 2$-modular left ideal of $R$. Now $R / T$ is an $R$-group of type-5/2. Since $T$ is modular, by Corollary 3.24 of [3], we get that $(T: R) \subseteq T$. So $\mathrm{J}_{5 / 2}(R) \subseteq$ $(T: R) \subseteq T$. Hence $\mathrm{J}_{5 / 2}(R)$ is the intersection of all $5 / 2$-modular left ideals of $R$.

Lemma 3.5. Let $R$ be a zero-symmetric near-ring and $S$ be an invariant subnearring of $R$. If $L$ is a 5/2-modular left ideal of $S$, then $L$ is an ideal of the $R$-group $S$ and $S / L$ is an $R$-group of type-5/2.

Proof. Let $L$ be a $5 / 2$-modular left ideal of $S$. Since an $R$-group of type$5 / 2$ is an $R$-group of type-2, $L$ is a 2-modular left ideal of $S$. Therefore, by Theorem 3.34 of [3], $L$ is an ideal of the $R$-group $S$ and $S / L$ is an $R$ group of type-2. Let $0 \neq s+L \in S / L$. Since $S / L$ is an $S$-group of type$5 / 2, S(s+L)=S / L$. Therefore $S / L=S(s+L) \subseteq R(s+L) \subseteq S / L$. So $R(s+L)=S / L$ and hence $S / L$ is an $R$-group of type-5/2.

Theorem 3.6. Let $S$ be an invariant subnear-ring of a zero-symmetric nearring $R$. Then $\mathrm{J}_{5 / 2}(S) \subseteq \mathrm{J}_{5 / 2}(R) \cap S$.

Proof. If $S$ has no 5/2-primitive ideals then $\mathrm{J}_{5 / 2}(S)=S \subseteq \mathrm{J}_{5 / 2}(R) \cap S$. So, suppose that $S$ has 5/2-primitive ideals. Let $P$ be a 5/2-primitive ideal of $S$. We get an $S$-group $G$ of type-5/2 such that $P=(0: G)_{S}$. Let $0 \neq g \in G$. Now $S /(0: g)_{S}$ and $G$ are isomorphic as $S$-groups and that $L:=(0: g)_{S}$ is a $5 / 2$-modular left ideal of $S$ and $P=(0: G)_{S}=(0: S / L)_{S}=(L: S)_{S}$. By Lemma $3.5, S / L$ is an $R$-group of type-5/2. So $Q:=(0: S / L)_{R}=(L: S)_{R}$ is a 5/2-primitive ideal of $R$. Therefore $P=(L: S)_{S}=(L: S)_{R} \cap S=Q \cap S$. Hence $\mathrm{J}_{5 / 2}(S) \subseteq \mathrm{J}_{5 / 2}(R) \cap S$.

Lemma 3.7. Let $S$ be an invariant subnear-ring of a zero-symmetric nearring $R$. Let $L$ be a 5/2-modular left ideal of $R$ and $S \nsubseteq L$. Then $L \cap S$ is a 5/2-modular left ideal of $S$.

Proof. We have that $L$ is a $5 / 2$-modular left ideal of $R$ and $S \nsubseteq L$. Now $R=S+L$. So $R / L=(S+L) / L \simeq_{R} S /(S \cap L)$ and that $S /(S \cap L)$ is an $R$-group of type-5/2. Let $L$ be modular by $e$. Now $r-r e \in L$ for all $r \in R$. Let $s \in S-(S \cap L)$. Since $0 \neq s+L \in R / L, R(s+L)=R / L$ and that $R s+L=R$. 
Now $e=r s+l, r \in R, l \in L . S \cap L$ is a left ideal of $S$ modular by $r s$. Let $t \in S$. Now $t e-t \in L$. So $t e-t=t(r s+l)-t=(t(r s+l)-t(r s))+(t(r s)-t) \in L$ and that $t(r s)-t \in L \cap S$. Therefore $t+(L \cap S)=t(r s)+(L \cap S) \in(S s+L \cap S) /(L \cap S)$ and that $S /(L \cap S)=(S s+L \cap S) /(L \cap S)=S(s+(L \cap S)$. Hence $S /(L \cap S)$ is an $S$-group of type-5/2. Since $L \cap S$ is a modular left ideal of $S, L \cap S$ is a $5 / 2$-modular left ideal of $S$.

Theorem 3.8. Let $R$ be a zero-symmetric near-ring and $S$ be an invariant subnearring of $R$. Then $\mathrm{J}_{5 / 2}(S) \subseteq \mathrm{J}_{5 / 2}(R) \cap S$.

Proof. If $\mathrm{J}_{5 / 2}(R)=R$, then $\mathrm{J}_{5 / 2}(S) \subseteq R \cap S=\mathrm{J}_{5 / 2}(R) \cap S$. Suppose that $\mathrm{J}_{5 / 2}(R) \neq R$. So $R$ has $5 / 2$-modular left ideals. Let $L$ be a $5 / 2$-modular left ideal of $R$. If $S \subseteq L$, then $\mathrm{J}_{5 / 2}(S) \subseteq S \cap L$. Now suppose that $S \nsubseteq L$. By Lemma 3.7, $S \cap L$ is a $5 / 2$-modular left ideal of $S$. So $\mathrm{J}_{5 / 2}(\mathrm{~S}) \subseteq S \cap L$. Therefore, by Proposition 3.4, $\mathrm{J}_{5 / 2}(S) \subseteq \mathrm{J}_{5 / 2}(R) \cap S$.

Theorem 3.9. Let $R$ be a zero-symmetric near-ring and $S$ be an invariant subnearring of $R$. Then $\mathrm{J}_{5 / 2}(S)=\mathrm{J}_{5 / 2}(R) \cap S$.

Theorem 3.10. $\mathrm{J}_{5 / 2}$ is an ideal-hereditary Kurosh-Amitsur radical in the class of all zero-symmetric near-rings.

We show now that $\mathrm{J}_{5 / 2}$ is not a KA-radical in the class of all near-rings.

Consider the dihedral group $\mathrm{D}_{8}=\{0, a, 2 a, 3 a, b, a+b, 2 a+b, 3 a+b\}$. Let $T$ be the near-ring given in Example 11 of $[3],($ p.418) whose additive group is $D_{8}$. As mentioned in [4], $\{0\}, J=\{0, a, 2 a, 3 a\}$ and $T$ are the ideals of $T$. Moreover, these are the only left ideals of $T$. Now $T / J$ is the constant near-ring on $\mathrm{Z}_{2}$. Since $T / J$ is a $T$-group of type-5/2, $J$ is a $5 / 2$-primitive ideal and is the only $5 / 2$-primitive ideal of $T$. So $\mathrm{J}_{5 / 2}(T)=J$.

We need the following result.

Proposition 3.11 (Proposition 3.3 of [4]). Let $Q$ be an ideal-mapping which satisfies ( $\mathrm{H} 1)$ and for which $Q(T)=J$ and $F \in S_{Q}$, where $F$ is the field of order 2. Then $Q$ is not idempotent and hence not a KA-radical mapping.

Theorem 3.12. $\mathrm{J}_{5 / 2}$ is not a $\mathrm{KA}$-radical in the class of all near-rings.

Proof. By Proposition 3.3, we have that $\mathrm{J}_{5 / 2}$ is the H-radical corresponding to the class of all 5/2-primitive near-rings. As seen above $\mathrm{J}_{5 / 2}(T)=J$. Moreover, the two element field is in $\mathcal{S}_{\mathrm{J}_{5 / 2}}$. So, by Proposition $3.11, \mathrm{~J}_{5 / 2}$ is not a KAradical in the class of all near-rings.

\section{The Jacobson radical of type- $(5 / 2)(0)$}

It is known that Jacobson radicals of type- 2 and 3 are ideal-hereditary KAradicals in the class of all zero-symmetric near-rings and the Jacobson radical of type-2 is not even a KA-radical in the class of all near-rings. S. Veldsman [5] introduced $R$-groups of type-2(0) and $3(0)$ and the corresponding Jacobson 
radicals of type-2(0) and $3(0)$ which are extensions of the Jacobson radicals of type- 2 and 3 respectively of zero-symmetric near-rings to the class of all nearrings and has shown that these two new radicals are KA-radicals in the class of all near-rings.

In this section we introduce $R$-groups of type- $(5 / 2)(0)$ and the corresponding Jacobson radical of type- $(5 / 2)(0)$. We show that it is a KA-radical in the class of all near-rings.

Definition 4.1. Let $G$ be an $R$-group of type-5/2. $G$ is called an $R$-group of type- $(5 / 2)(0)$ if $R 0=\{0\}$, where 0 is the additive identity in $G$.

Proposition 4.2. Let $G$ be an R-group of type-5/2. Then $G$ is an $R$-group of type-(5/2)(0) if and only if $R_{c} \subseteq(0: G)$, where $R_{c}$ is the constant part of $R$.

Proof. Let $G$ be an $R$-group of type-(5/2)(0). $R_{c} g=(R o) g=R(o g)=R 0=$ $\{0\}$ for all $g \in R$. So, $R_{c} \subseteq(0: G)$. Suppose now that $R_{c} \subseteq(0: G)$. Now $R_{c} 0$ $=\{0\}$, where 0 is the additive identity in $G$. So $R 0=\{0\}$ and hence $G$ is an $R$-group of type- $(5 / 2)(0)$.

Corollary 4.3. Let $R$ is a zero-symmetric near-ring and $G$ be an $R$-group. Then $G$ is type- $(5 / 2)(0)$ if and only if it is of type-5/2.

Definition 4.4. A near-ring $R$ is said to be $(5 / 2)(0)$-primitive if it has a faithful $R$-group of type-(5/2)(0). An ideal $I$ of $R$ is called (5/2)(0)-primitive if $R / I$ is a $(5 / 2)(0)$-primitive near-ring.

Proposition 4.5. Let $I$ be an ideal of $R$. Then the following are equivalent.

(i) $I$ is $(5 / 2)(0)$-primitive ideal of $R$.

(ii) $I=(0: G)$ for some $R$-group $G$ of type- $(5 / 2)(0)$.

Proof. Suppose that $I$ is a $(5 / 2)(0)$-primitive ideal of $R . R / I$ is a $(5 / 2)(0)$ primitive on some $R / I$-group $G$ of type- $(5 / 2)(0)$. Since $G$ is a faithful $R / I$ group of type- $(5 / 2)(0), G$ is an $R$-group of type-5/2 and $I=(0: G)$. Also, since $R / I$ is zero-symmetric, $R_{c} \subseteq I=(0: G)$ and hence $G$ is an $R$-group of type-(5/2)(0). Conversely, suppose that $I=(0: G)$ for an $R$-group $G$ of type- $(5 / 2)(0)$. Since $G$ is an $R$-group of type- $(5 / 2)(0)$ and $I=(0: G), G$ is a faithful $R / I$-group of type-5/2. Also since $\mathrm{R}_{c} \subseteq(0: G)=I, R / I$ is a zerosymmetric near-ring and hence $G$ is a faithful $R / I$-group of type- $(5 / 2)(0)$. So $R / I$ is a $(5 / 2)(0)$-primitive near-ring and hence $I$ is a $(5 / 2)(0)$-primitive ideal of $R$.

Corollary 4.6. The following are equivalent

(i) $\{0\}$ is a $(5 / 2)(0)$-primitive ideal of $R$.

(ii) $R$ is (5/2)(0)-primitive.

Corollary 4.7. $R$ is $(5 / 2)(0)$-primitive if and only if $R$ is a zero-symmetric and $(5 / 2)$-primitive. 
Remark 4.8. It is clear that a $(5 / 2)(0)$-primitive ideal of $R$ contains $R_{c}$, the constat part of $R$.

Definition 4.9. Let $R$ be a near-ring. $J_{(5 / 2)(0)}(R)$ is defined as the intersection of all (5/2)(0)-primitive ideal of $R$ and $\mathrm{J}_{(5 / 2)(0)}(R)=R$ if $R$ has no (5/2)(0)primitive ideals. $\mathrm{J}_{(5 / 2)(0)}$ is called the Jacobson radical of type-(5/2)(0).

Remark 4.10. If $R$ is a ring, then $\mathrm{J}_{(5 / 2)(0)}(R)$ is the Jacobson radical of $R$.

We show now that $J_{(5 / 2)(0)}$ is a KA-radical in the class of all near-rings, its semisimple class is hereditary and radical class is $c$-hereditary.

Theorem 4.11. The class of all zero-symmetric 5/2-primitive near-rings satisfy condition $\left(F_{l}\right)$.

Proof. Since a zero-symmetric 5/2-primitive near-ring is a 2-primitive nearring, by Theorem 1.2, we get that the class of all zero-symmetric 5/2-primitive near-rings also satisfy condition $\left(\mathrm{F}_{l}\right)$.

Theorem 4.12. Let $R$ be a near-ring. $\mathrm{J}_{(5 / 2)(0)}$ is a KA-radical in the class of all near-rings, $\mathrm{J}_{(5 / 2)(0)}(I) \subseteq \mathrm{J}_{(5 / 2)(0)}(R) \cap I$ for all $I \triangleleft R$ and the equality holds if $I$ is a left invariant ideal.

Proof. Let $\mathbb{M}$ be the class of all zero-symmetric $5 / 2$-primitive near-rings. Now by Corollary $4.7, \mathrm{~J}_{(5 / 2)(0)}(R)=(R) \mathbb{M}$ for all near-rings $R$. By Theorem 3.10 , $\mathrm{J}_{5 / 2}$ is an ideal-hereditary $\mathrm{KA}$-radical in the class of all zero-symmetric nearrings. In view of Theorem 1.1, it is enough to show that $M$ satisfies condition $(*)$ of Theorem 1.1. Let $K \triangleleft I \triangleleft R$ and $I$ be a left invariant ideal of $R$ with $I / K \in \mathbb{M}$. By Theorem 4.11, M satisfies condition $\left(F_{l}\right)$. So $K \triangleleft R$. Since $I$ is a left invariant ideal of $R, R_{c} \subseteq I$. Also since $I / K$ is a zero-symmetric near-ring, $R_{c}=I_{c} \subseteq K$. Since $R_{c} \subseteq K$ and $K \triangleleft R$, we get that $\overline{R_{c}} \subseteq K$. This completes the proof.

\section{References}

[1] G. Betsch, Struktursätze für Fastringe, Inaugural-Dissertation zur Erlangung des Grades eines Doktors der Naturwissenschaften der Mathematisch-Naturwissenschaftlichen Fakultät der Eberhard-Karls-Universität zu Tübingen Paul Jllg, Photo-Offsetdruck, Stuttgart 1963.

[2] M. Holcombe, A hereditary radical for near-rings, Studia Sci. Math. Hungar. 17 (1982), no. $1-4,453-456$.

[3] G. Pilz, Near-Rings: The Theory and Its Applications, Second edition. North-Holland Mathematics Studies, 23. North-Holland Publishing Co., Amsterdam, 1983.

[4] S. Veldsman, On the nonhereditariness of radical and semisimple classes of near-rings, Studia Sci. Math. Hungar. 24 (1989), no. 2-3, 315-323.

[5] _ Modulo-constant ideal-hereditary radicals of near-rings, Quaestiones Math. 11 (1988), no. 3, 253-278. 


\author{
RAVI SRINIVASa RaO \\ Department of Mathematics \\ P. G. CENTRE \\ P. B. Siddhartha College of ARts and Science \\ ViJaYaWada-520010, ANdhra Pradesh, INDIA \\ E-mail address: dr_rsrao@yahoo.com \\ K. Siva PRAsad \\ DEPARTMENT OF MATHEMATICS \\ Chalapathi Institute of Engineering and Technology \\ Chalapathi Nagar, Lam \\ Guntur-522034, Andhra Pradesh, India
}

